\title{
Kinetics and Energetics of Azo Dye Decolorization by Pycnoporus sanguineus
}

\author{
M. S. M. Annuar • S. Adnan • S. Vikineswary • \\ Y. Chisti
}

Received: 4 September 2008 / Accepted: 22 December 2008 / Published online: 16 January 2009

(C) Springer Science + Business Media B.V. 2009

\begin{abstract}
Trypan Blue, an azo dye, was decolorized using the self-immobilizing fungal biomass of Pycnoporus sanguineus. The extent and the rate of dye decolorization were directly proportional to the initial dye concentration $\left(20-60 \mathrm{mg} \mathrm{L}^{-1}\right)$ and the reaction temperature $\left(25-45^{\circ} \mathrm{C}\right)$. Mass transfer within and outside the pellets did not limit dye degradation. The apparent kinetics of the decolorization reaction followed a first-order behavior. Activation energy for the biological decolorization was calculated at $23 \mathrm{~kJ}$ $\mathrm{mol}^{-1}$. The decolorization process was endothermic with the enthalpy and entropy values calculated at $45.6 \mathrm{~kJ} \mathrm{~mol}^{-1}$ and $146 \mathrm{~J} \mathrm{~mol}^{-1} \mathrm{~K}^{-1}$, respectively. Based on the value of Gibbs free energy change, the decolorization reaction under the conditions studied was non-spontaneous below $39^{\circ} \mathrm{C}$ but was spontaneous at higher temperatures.
\end{abstract}

Keywords Dye decolorization .

Pycnoporus sanguineus · Kinetics · Energetics ·

Fungal pellets

M. S. M. Annuar $(\bowtie) \cdot S$. Adnan $\cdot$ S. Vikineswary Institute of Biological Sciences, Faculty of Science, University of Malaya,

50603 Kuala Lumpur, Malaysia

e-mail: suffian_annuar@um.edu.my

Y. Chisti

School of Engineering, PN 456, Massey University,

Private Bag 11 222,

Palmerston North, New Zealand

\section{Introduction}

Synthetic dyes are widely used in industrial processes. More than $7.0 \times 10^{5}$ tons of dyes are produced annually worldwide. A significant amount of this material is lost in various dyeing operations. For example, almost $10 \%$ to $15 \%$ of the total dyes consumed in the textile industry are lost during dyeing (Zollinger 1987). Consequently, large quantities of dye-containing effluents are released in the environment. Such effluents discolor waterbodies and increase biochemical oxygen demand of the contaminated water. In addition, anaerobic degradation products of some dyes may be carcinogenic or mutagenic (Shin et al. 2002).

Treatment of dye effluent often involves both physical and chemical methods that are expensive and not always effective. Therefore, extensive effort is being directed to development of inexpensive and environmentally friendly bioremediation methods. Synthetic dyes have been shown to be decolorized by white-rot fungi. The non-specific extracellular lignin-modifying enzymes (LMEs) of these fungi are responsible for degrading the dyes (Pointing 2001).

The white-rot fungi and their bulk LMEs can be produced by inexpensive fermentation processes. This makes dye bioremediation cost effective. Under favorable conditions in submerged fermentation, the white-rot fungi can self-immobilize into spherical pellets and this facilitates biomass removal from LME-containing broth. Both the pellets and the 
biomass-free LME-containing broth are potentially effective in decolorizing dye-contaminated wastewaters.

This work reports on the kinetics and bio-energetics of decolorization of Trypan Blue, an azo dye, by spherical pellets of the white-rot fungus Pycnoporus sanguineus. The water-soluble Trypan Blue was used as a general model for the azo class dyes.

\section{Materials and Methods}

\subsection{Microorganism and Growth Medium}

The white-rot fungus Pycnoporus sanguineus Linn. Ex Fr (Murrill) was maintained on potato dextrose agar (PDA) at $4^{\circ} \mathrm{C}$. The fungus was sub-cultured on PDA plates and incubated at $27^{\circ} \mathrm{C}$ for a week prior to use in the experiments.

Glucose-yeast extract-malt extract-peptone (GYMP) medium was used to grow the fungus in liquid suspensions. The composition of the medium was (g L $\left.{ }^{-1}\right)$ : $0.5 \mathrm{MgSO}_{4}, 1.0 \mathrm{~K}_{2} \mathrm{HPO}_{4}, 0.46 \mathrm{KH}_{2} \mathrm{PO}_{4}$, 20.0 glucose, 2.0 peptone (Becton-Dickinson), 2.0 yeast extract (Becton-Dickinson), and 2.0 malt extract (Becton-Dickinson). The medium without the glucose was sterilized by autoclaving at $121^{\circ} \mathrm{C}$ for $15 \mathrm{~min}$. Glucose solution was sterilized separately $\left(121^{\circ} \mathrm{C}\right.$, $15 \mathrm{~min}$ ), cooled to room temperature, and then added aseptically to the rest of the medium at room temperature.

\subsection{Growth and Preparation of Fungal Pellets}

The fungus that had been sub-cultured on PDA plates was used to prepare the biomass pellets in the liquid medium identified above. Approximately $10 \mathrm{~mL}$ of a $0.1 \%$ aqueous solution of polyoxyethylene sorbitan monooleate (Tween 80) were pipetted on to the mycelium mat on the surface of a Petri dish. The mycelium was scraped using a sterile loop and transferred to a MacCartney bottle that contained
$20 \mathrm{~mL}$ of $0.1 \%$ Tween 80 . This suspension was mixed vigorously using a vortex mixer. A $2.0-\mathrm{mL}$ aliquot of this suspension was inoculated into $100 \mathrm{~mL}$ of GYMP liquid medium in a $250-\mathrm{mL}$ Erlenmeyer flask that was incubated on a rotary shaker for 4 days $\left(27 \pm 1^{\circ} \mathrm{C}, 150 \mathrm{rpm}\right)$. Several flasks were started in parallel. The suspension of pellets produced was filtered using Whatman No. 1 filter paper to recover the spherical pellets. The retained pellets were rinsed with $300 \mathrm{~mL}$ of distilled water and then dried at room temperature to constant weight which was then recorded. The drying process took approximately $12 \mathrm{~h}$. The pellets were used immediately. The average diameter of freshly harvested pellets was calculated based on measurements on several batches of 50 pellets each.

\subsection{Synthetic Dye and Concentration Measurements}

Trypan Blue $\left(\mathrm{C}_{34} \mathrm{H}_{24} \mathrm{~N}_{6} \mathrm{O}_{14} \mathrm{~S}_{4} \mathrm{Na}_{4}\right.$; molar mass of 961 Da; Fig. 1) (Sigma, St. Louis, MO, USA), a water-soluble synthetic dye, was used as a model azo dye in the decolorization study. A stock solution of the dye was prepared in distilled water at a concentration of $1,000 \mathrm{mg} \mathrm{L}^{-1}$. This solution was diluted to appropriate concentrations before use. The stock solution was stored in an amber bottle to protect it from direct sunlight and kept at $4^{\circ} \mathrm{C}$.

Concentration of the dye was routinely measured spectrophotometrically at $597 \mathrm{~nm}$. The absorbance was converted to concentration using a calibration curve that fitted the following equation:

$C_{\text {dye }}=31.847 A_{597}$

where $C_{\text {dye }}$ was the concentration of the dye in $\mathrm{mg}$ $\mathrm{L}^{-1}$ and $A_{597}$ was the absorbance of the solution at $597 \mathrm{~nm}$. Equation (1) had a regression coefficient of 0.996 and applied over a concentration range from 0 to $30 \mathrm{mg} \mathrm{L}^{-1}$.

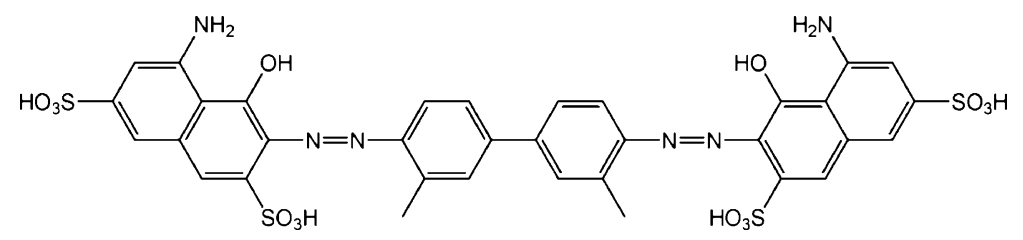

Fig. 1 Structure of Trypan Blue 


\subsection{Dye Decolorization by Fungal Pellets}

Dried fungal pellets $(3 \pm 0.1 \mathrm{~g}, \sim 20$ pellets $)$ were transferred to a $250-\mathrm{mL}$ Erlenmeyer flask that contained $100 \mathrm{~mL}$ of dye solution at some predetermined initial concentration $(20,40$, and $60 \mathrm{mg}$ $\left.\mathrm{L}^{-1}\right)$. The flasks were incubated on a rotary shaker at $100 \mathrm{rpm}$ for $24 \mathrm{~h}$. Incubation temperatures of 25, 30, 35,40 , and $45^{\circ} \mathrm{C}$ were used in different experiments. The solution in the flasks was sampled at regular intervals for measurement of the dye concentration. At the end of the incubation period (i.e., $24 \mathrm{~h}$ ), the pellets were harvested by filtration as above, washed with $150 \mathrm{~mL}$ distilled water, and extracted with $150 \mathrm{~mL} \mathrm{95 \%} \mathrm{ethanol} \mathrm{to} \mathrm{recover} \mathrm{any} \mathrm{dye} \mathrm{adsorbed} \mathrm{to}$ the pellets. All experiments were in triplicate. Each sampling was also in triplicate. Control experiments were conducted in parallel under the above identified conditions (dye concentration, agitation speed, and temperature), but without the biomass pellets.

\subsection{Dye Decolorization by Biomass-free Filtrate} of the Spent Culture Medium

The biomass-free culture filtrate obtained at the end of the pellet production stage was assessed for its dye decolorization activity. The filtrate was mixed with a dye solution at a volume ratio of $1: 1$ and incubated for $24 \mathrm{~h}$ in $250-\mathrm{mL}$ shake flasks at $100 \mathrm{rpm}$. Different dye concentrations and incubation temperatures were used in different experiments. Flasks were sampled periodically for spectrophotometric measurement of the dye concentration. The biomass-free culture filtrate was also tested for laccase enzyme activity.

2.6 Dye Decolorization in Biomass-free Dye Solution Pre-incubated with Fungal Pellets

Dried fungal pellets were added to the dye solution and incubated for $24 \mathrm{~h}$ under specified conditions. Subsequently, the pellets were removed by filtration and the biomass-free dye solution was further incubated for $24 \mathrm{~h}$. Samples were taken periodically and measured for the dye concentration as specified above.

\subsection{Adsorbed Dye Decolorization by Fungal Biomass}

To test if Trypan Blue adsorbed on fungal pellets would be decolorized, a known amount of fungal pellets was immersed in a dye solution for $24 \mathrm{~h}$. Then, the blue-colored pellets were removed by filtration, rinsed with $100 \mathrm{~mL}$ of distilled water, and further incubated in a closed, dry beaker for $24 \mathrm{~h}$. Significant decolorization, if any, was assessed by visual inspection. Pellets that had been boiled in water $(20 \mathrm{~min})$ were used as controls. The boiled pellets were subjected to the same treatment as the dried pellets.

\subsection{Assay for Laccase Enzyme Activity}

The enzyme substrate used in this assay was syringaldazine (4-hydroxy-3,5-methoxybenzaldehyde azine; Sigma). A 0.1-mM solution of syringaldazine was prepared by dissolving it in 50\% aqueous ethanol while stirring for $13 \mathrm{~h}$. This solution was stored at $4^{\circ} \mathrm{C}$ until needed. Before use, the solution was warmed to room temperature.

For measuring the laccase activity, a $0.2-\mathrm{mL}$ biomass-free sample of the culture filtrate was mixed with $3.0 \mathrm{~mL}$ of $50 \mathrm{mM}$ sodium citrate $(\mathrm{pH} 4.8)$ buffer at room temperature. Then $0.2 \mathrm{~mL}$ of $0.1 \mathrm{mM}$ syringaldazine was added and mixed gently. The total volume of the reaction mixture was $3.4 \mathrm{~mL}$. The spectrophotometric absorbance was measured immediately at $525 \mathrm{~nm}$ and again after incubation (room temperature) for $10 \mathrm{~min}$.

Laccase activity $(\mathrm{U} / \mathrm{L})=\frac{\Delta \mathrm{Abs}}{\Delta t \varepsilon l} \times \frac{\text { total assay volume }}{\text { enzyme sample volume }}$

where $\Delta \mathrm{Abs}$ is the change in absorbance, $\Delta t$ is the time of incubation ( $\mathrm{min}), \varepsilon$ is the extinction coefficient of syringaldazine at $525 \mathrm{~nm}\left(65,000 \mathrm{M}^{-1} \mathrm{~cm}^{-1}\right)$, and $l$ is the cuvette diameter $(1 \mathrm{~cm})$. One unit of enzyme activity was defined as the amount of enzyme that oxidized $1 \mu \mathrm{mol}$ of syringaldazine per minute. Activity was measured in triplicate.

\section{Calculations}

\subsection{Trypan Blue Decolorization}

The actual amount of the dye decolorized was calculated based on a mass balance, as follows:

$M_{\text {decolorized }}=M_{\text {initial }}-M_{\text {residual }}-M_{\text {adsorbed }}$ 
where $M_{\text {decolorized }}$ is the mass of Trypan Blue dye decolorized, $M_{\text {initial }}$ is the initial mass of the dye, $M_{\text {residual }}$ is the mass of the dye remaining in solution, and $M_{\text {adsorbed }}$ is the mass of the dye adsorbed by the pellets.

\subsection{Rate of Dye Decolorization}

Volumetric rate of dye decolorization $\left(r_{\mathrm{vol}} ; \mathrm{mg} \mathrm{L}^{-1} \mathrm{~h}^{-1}\right)$ was calculated according to the following equation:

$r_{\mathrm{vol}}=\frac{\Delta C(t)}{\Delta t}$

where $\Delta C(t)\left(\mathrm{mg} \mathrm{L}^{-1}\right)$ is the change in dye concentration over the time interval $\Delta t(\mathrm{~h})$.

\subsection{External Mass Transfer}

Observable modulus for external mass transfer, $\Omega$, for a pellet was calculated as follows:

$\Omega=\frac{R}{3}\left(\frac{r_{\mathrm{obs}}}{K_{S} C_{\mathrm{bulk}}}\right)$

where $R$ is the average radius of pellet (m), $r_{\mathrm{obs}}$ is the rate of dye consumption per unit volume of pellets at steady state $\left(\mathrm{g} \mathrm{m}^{-3} \mathrm{~s}^{-1}\right), K_{S}$ is the liquid-phase mass transfer coefficient $\left(\mathrm{m} \mathrm{s}^{-1}\right)$, and $C_{\text {bulk }}$ is the dye concentration in the bulk liquid $\left(\mathrm{g} \mathrm{m}^{-3}\right)$. Because no data was available for the liquid-phase mass transfer coefficient of Trypan Blue, the $K_{S}$ value for phenol in water (Vinod and Reddy 2006) was used in Eq. (5) as being representative for Trypan Blue. This $K_{S}$ value was $20.12 \times 10^{-7} \mathrm{~m} \mathrm{~s}^{-1}$.

\subsection{Dye Concentration Profile in the Pellet}

Assuming a uniform distribution of the dye-degrading enzyme in the porous mycelial pellet, a spherical shape of the pellet, isothermal conditions, a first-order reaction and no partitioning of the substrate between the exterior and interior of the pellet, and the rate of diffusion of the dye in the pellet at steady state was equated to the rate of the consumption as follows:

$D_{e} \frac{\mathrm{d}^{2} C_{\text {dye }}}{\mathrm{d} r^{2}}+\frac{2}{r} \frac{\mathrm{d} C_{\text {dye }}}{\mathrm{d} r}=k_{1}^{\prime} C_{\text {dye }}$

In Eq. (6), $C_{\text {dye }}$ is the Trypan Blue concentration within the pellet $\left(\mathrm{g} \mathrm{m}^{-3}\right), r$ is the radial position in the pellet $(\mathrm{m}), k_{1}^{\prime}$ is the apparent first-order rate constant of dye decolorization $\left(\mathrm{h}^{-1}\right)$, and $D_{e}$ is the effective diffusion coefficient of the dye in the pellet $\left(\mathrm{m}^{2} \mathrm{~s}^{-1}\right)$. In the absence of a $D_{e}$ value for the system under investigation, the average $D_{e}$ value for phenol in Caalginate beads was used. This value was $1.21 \times$ $10^{-11} \mathrm{~m}^{2} \mathrm{~s}^{-1}$ (Dursun and Tepe 2005).

The boundary conditions used for solving Eq. (6) were: $C_{\text {dye }}=C_{\text {dye,s }}$ at $r=R$ and $\mathrm{d}_{\text {dye }} / \mathrm{d} r=0$ at $r=0$, where $R$ is the radius of the pellet and $C_{\mathrm{dye}, \mathrm{s}}$ is the concentration of dye at the outer surface of the pellet. Assuming the absence of an external mass transfer limitation, $C_{\text {dye,s }}$ is equal to the bulk concentration of dye, $C_{\text {dye }}$ (i.e., $C_{\text {dye,s }}=C_{\text {dye }}$ ). Integration of Eq. (6) gives the following equation:

$C_{\mathrm{dye}}=C_{\mathrm{dye}, \mathrm{s}} \frac{\sinh \left(r \sqrt{\left(k_{1}^{\prime} / D_{e}\right)}\right)}{\left(\frac{r}{R}\right) \sinh \left(R \sqrt{\left(k_{1}^{\prime} / D_{e}\right)}\right)}$

Equation (7) allows the estimation dye concentration within the pellet as a function of the local radial position $r$.

For estimating the apparent first-order rate constant $\left(k_{1}^{\prime}\right)$ for the decolorization process, the volumetric rate of dye degradation (Eq. 4) was plotted against the various initial dye concentrations. The rate constant $k_{1}^{\prime}$ $\left(\mathrm{h}^{-1}\right)$ was calculated as the slope of the resulting linear plot.

\subsection{Activation Energy}

The apparent activation energy $\left(E_{\mathrm{a}}, \mathrm{J} \mathrm{mol}^{-1}\right)$ of the decolorization process was estimated using the linearized Arrhenius equation:

$\ln k_{1}^{\prime}=\ln A-\frac{E_{\mathrm{a}}}{R T}$

where $A$ is the frequency factor, $R$ is the gas constant (8.3145 $\mathrm{J} \mathrm{mol}^{-1} \mathrm{~K}^{-1}$ ), and $T$ is the absolute temperature $(\mathrm{K})$. The values of $k_{1}^{\prime}$ measured at different temperatures were plotted against the reciprocal of the temperature and $E_{\mathrm{a}}$ was obtained from the slope of the resulting plot.

\subsection{Energetics Parameters}

The enthalpy and entropy of the dye decolorization process were determined using van't Hoff analysis. 
The degradation process was assumed to be at an equilibrium state when the residual dye concentration in solution no longer changed with time. At equilibrium,

$K_{\mathrm{eq}}=\frac{[P]_{\mathrm{eq}}}{[S]_{\mathrm{eq}}}$

where $K_{\text {eq }}$ is the apparent equilibrium constant, $[P]_{\text {eq }}$ is the concentration of dye that has been decolorized at equilibrium $\left([P]_{\mathrm{eq}}=\right.$ initial dye concentration - dye concentration remaining at equilibrium), and $[S]_{\mathrm{eq}}$ is the dye concentration remaining at equilibrium.

The apparent equilibrium constant $K_{\text {eq }}$ was measured at various temperatures. The temperature dependence of $K_{\text {eq }}$ is expressed according to van't Hoff equation as follows:

$\ln K_{\mathrm{eq}}=-\frac{\Delta H}{R T}+\frac{\Delta S}{R}$

where $\Delta H$ is van't Hoff enthalpy $\left(\mathrm{J} \mathrm{mol}^{-1}\right)$ and $\Delta S$ is the entropy $\left(\mathrm{J} \mathrm{mol}^{-1} \mathrm{~K}^{-1}\right)$.

The above analysis assumes that the decolorization reaction occurs in an aqueous medium at a constant pressure without changes in volume. Hence, $\Delta H$ represents the heat transferred to a system at constant pressure.

At constant temperature and pressure, Gibbs free energy change $\left(\Delta G, \mathrm{~J} \mathrm{~mol}^{-1}\right)$ for the reaction at nonstandard conditions was calculated using the following equation:

$\Delta G=\Delta H-T \Delta S$

Gibbs free energy at standard condition $\left(\Delta G^{\circ}, \mathrm{J} \mathrm{mol}^{-1}\right)$ was calculated as follows:

$\Delta G^{\circ}=\Delta G-R T \ln K_{\mathrm{eq}}$

where $K_{\text {eq }}$ is the apparent equilibrium constant at standard conditions.

\section{Results and Discussion}

\subsection{Effect of Initial Dye Concentration and Temperature}

Measurements on several batches of 50 pellets each revealed the average fresh weight of a pellet to be $0.015 \pm 0.005 \mathrm{~g}$. The average measured pellet diameter was $2.4 \pm 0.5 \mathrm{~mm}$. Each decolorization run involving pellets used $30 \pm 1 \mathrm{~g} \mathrm{~L}^{-1}$ of pellets ( $\sim 200$ pellets) equivalent to an approximate pellet volume of $1.64 \mathrm{~cm}^{3}$. The entire porous pellet was assumed to be catalytically active. The total mass of the catalyst, i.e., the pellets, within the total volume of the pellets was estimated to be $1.8 \mathrm{~g} \mathrm{~cm}^{-3}$.

The amount of Trypan Blue decolorized after $24 \mathrm{~h}$ incubation with the pellets at various temperatures is shown in Fig. 2, plotted against the initial concentration of the dye in solution. At any given temperature, the amount decolorized increased linearly with increasing initial concentration of the dye. At any given initial concentration, the extent of dye decolorization increased with increasing temperature. The observed degradation was ascribed to laccase activity as this is the sole phenoloxidase that is produced by $P$. sanguineus in submerged culture (Pointing and Vrijmoed 2000). P. sanguineus laccase has been previously shown to decolorize azo dyes such as Orange $G$ and Amaranth (Pointing and Vrijmoed 2000). The dye removal activity was quite stable at the highest temperature tested (Fig. 2). In all cases in Fig. 2, decolorization was visually complete after $24 \mathrm{~h}$. No visible growth of the fungus was observed during decolorization, as the medium used was devoid of nutrients. No decolorization occurred at any temperature in control experiments.

Based on the mass balance (Eq. 3), biological decolorization accounted for $70-90 \%$ of the dye removed. The remaining percentage was adsorbed on the fungal pellets and was decolorized eventually.

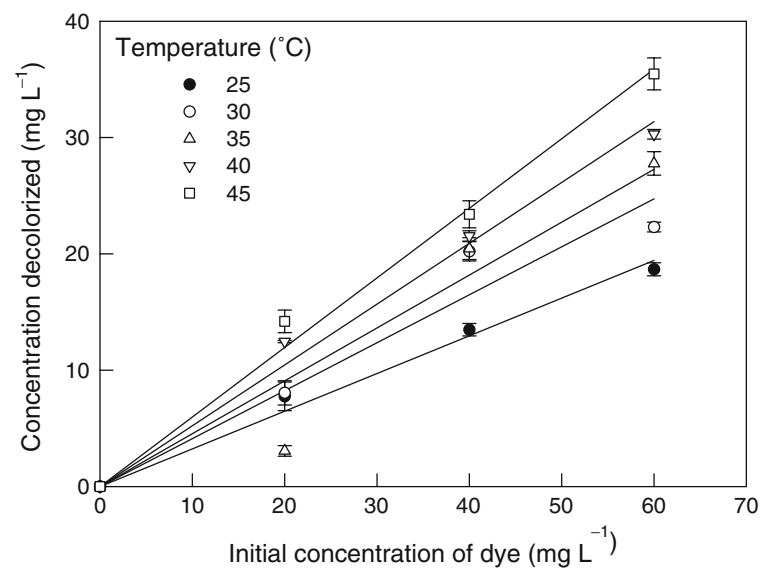

Fig. 2 Amount of Trypan Blue decolorized (24-h incubation) as a function of its initial concentration 
In studies with other azo and triphenylmethane dyes, sorption on the biomass has accounted for less than $3 \%$ of the dye removal by $P$. sanguineus (Pointing and Vrijmoed 2000). This suggests that the extent of dye adsorption by the biomass is different for different dyes.

\subsection{Dye Decolorization by Biomass-free Filtrate of the Spent Culture Medium}

The biomass-free filtrate obtained from fermentations that were used to grow the pellets was unable to decolorize Trypan Blue solutions. Furthermore, no laccase activity was detected in the culture filtrate. Clearly, all laccase produced by the pellets was within the biomass or bound to it. This is explained by the fact that the GYMP medium contained sufficient nutrients whereas nutrient limitation (e.g., nitrogen) is a key requirement for the expression of degradative enzymes by white-rot fungi (Field et al. 1992). Furthermore, the medium used did not contain any known inducer (e.g., phenolic compounds) of laccase. It is also suggested that exposure of the pellets to the dye solution could have induced a significant level of degradative enzyme(s) expression by the fungus.

\subsection{Dye Decolorization in Biomass-free Dye Solution Pre-incubated with Fungal Pellets}

No further decolorization of Trypan Blue was observed when pellets were removed from the dye solution after $24 \mathrm{~h}$ of incubation and the biomass-free dye solution was allowed to incubate for a further $24 \mathrm{~h}$. Clearly, all decolorizing activity was within the biomass, or on its surface, and did not enter the culture filtrate. The biomass pellets that were removed from the dye solution earlier than $24 \mathrm{~h}$ had a significant quantity of adsorbed blue coloration. On standing in a covered beaker for $24 \mathrm{~h}$, the pellets completely degraded the blue color. In control experiments, the dye did adsorb to the boiled pellets but the pellets were unable to decolorize the adsorbed dye. This further confirmed that all observed decolorization was due to enzymes that were inseparably linked to the biomass and did not leak into the culture filtrate. The observation that both viable and dead fungal pellets readily adsorbed the blue dye indicated a strong adsorption affinity between the pellets and the dye.
Significantly, all decolorization in this work was achieved without any addition of an external redox mediator or growth substrate such as glucose. Such additives have been shown to improve the rate of dye decolorization by white-rot fungi such as Trametes versicolor (Swamy and Ramsay 1999a).

\subsection{External Mass Transfer Limitation Analysis}

Analysis suggested that external mass transfer of the dye to the pellets posed no limitation on the decolorization kinetics in this work. This is evidenced by the low values of the mass transfer modulus $\Omega$ in Fig. 3. Since the $\Omega$ value was always much smaller than 1 , this clearly indicates that the difference between dye concentration at the surface of the pellets and its concentration in the bulk liquid was infinitesimally small. At the lowest temperature of $25^{\circ} \mathrm{C}$ and the lowest dye concentration of $20 \mathrm{mg} \mathrm{L}^{-1}$, the $\Omega$ value for the smallest pellets $\left(1.9 \times 10^{-3} \mathrm{~m}\right.$ in diameter) was 0.04 . Under the same conditions for the largest pellets $\left(2.9 \times 10^{-3} \mathrm{~m}\right.$ in diameter $)$, the $\Omega$ value was 0.07 . From Fig. 3, it is also observed that the transport rate is reduced at higher initial dye concentration. This is due to the fact that, at any particular temperature tested, the increased change in observed rate of reaction, $r_{\mathrm{obs}}$ (between $\Delta 0.2-04 \mathrm{mg}$ $\left.\mathrm{L}^{-1} \mathrm{~h}^{-1}\right)$, is much smaller than the increased change in initial dye concentration $\left(\Delta 20 \mathrm{mg} \mathrm{L}^{-1}\right)$. As a result, a lowered transport rate calculated by Eq. (5) was obtained.

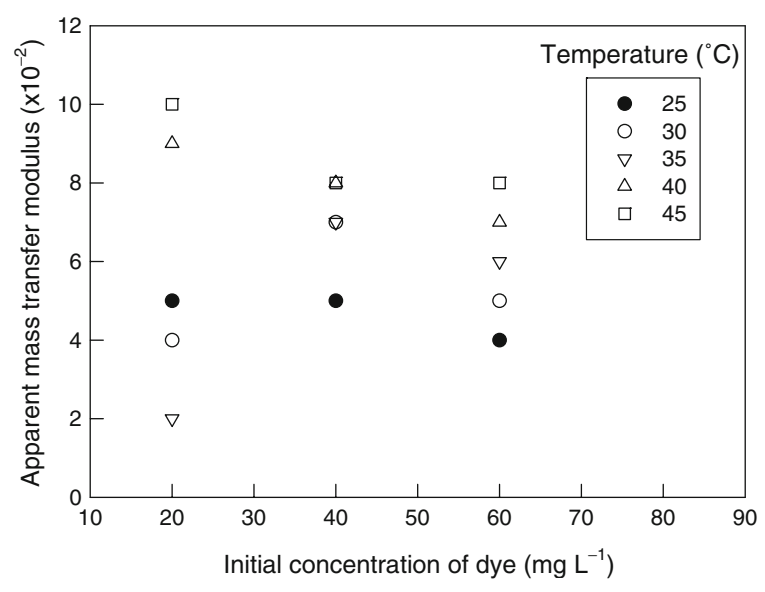

Fig. 3 Modulus for external mass transfer as a function of initial dye concentration 


\subsection{Concentration Profile within the Pellet}

The dye concentration profile within a pellet as a function of its radius was estimated using Eq. (7). This profile is shown in Fig. 4. There was only a slight drop in concentration of the dye in going from the surface of the pellet to its interior. In view of this small variation in concentration, the rate of decolorization within most of the pellet can be concluded to be nearly the same as at the pellet's surface. In a pellet with an average radius of $1.2 \mathrm{~mm}$, the calculated dye concentration at a distance of $0.02 \mathrm{~mm}$ from the center was only $8 \%$ lower than the concentration at the pellet's surface. Although the calculated concentration profile within the pellet showed that the dye concentration was zero at the center of the pellet (Fig. 4), in reality it is also very likely that the dye concentration at the center of the pellet may approach some finite value above zero. This is due to the observation that the dye was readily adsorbed by the pellets which to some extent may be attributed to the relatively porous structure of the individual pellet. Based on this analysis, mass transfer within the pellet was also suggested to pose no limitation on the rate of degradation.

Although the intraparticle mass transfer of the dye is not a significant limitation on degradation kinetics, mass transfer of molecular oxygen may pose a limitation. Laccase requires molecular oxygen as a cosubstrate in the monoelectronic oxidation of a suitable substrate molecule (e.g., phenols, aromatic or aliphatic amines) to the corresponding reactive radical. The overall outcome of the catalytic cycle is the reduction

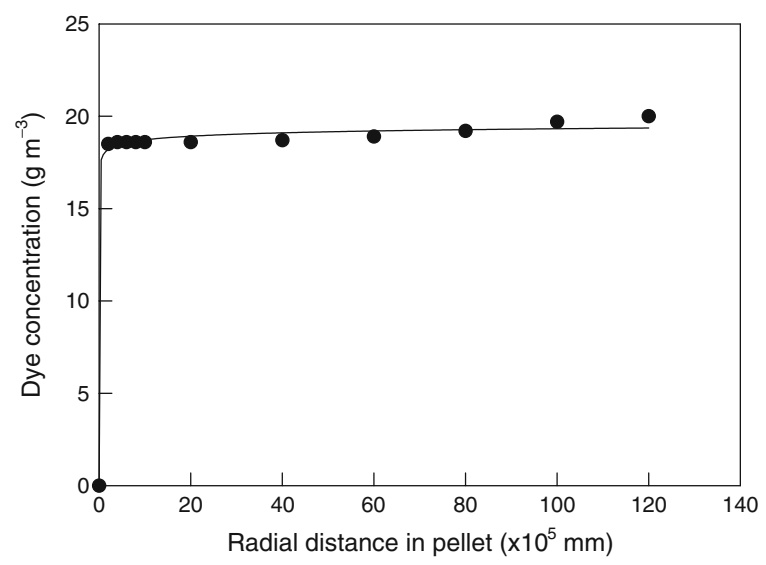

Fig. 4 Dye concentration profile within a pellet of one molecule of oxygen to two molecules of water and the concomitant oxidation of four substrate molecules to produce four radicals (Riva 2006). In pellet cultures of the white-rot fungus Phanerochaete chrysosporium, Michel et al. (1992) showed that in a pellet of $1.85 \mathrm{~mm}$ diameter, oxygen mass transfer was rate limiting. The concentration of oxygen became undetectable at a depth of less than $0.4 \mathrm{~mm}$ from the surface. This occurred even when the oxygen concentration at the pellet's surface was the same as in the culture fluid surrounding the pellet. In the event of a severe oxygen transfer limitation occurring within pellets, the maximum rate of Trypan Blue decolorization would be mostly confined to the surface of the pellets.

\subsection{Kinetics of Decolorization}

As shown in Fig. 5a-e, at any given temperature, the volumetric rate of dye decolorization increases linearly with increasing initial concentration of the dye. In view of these observations, the decolorization of Trypan Blue is hypothesized to follow the first-order reaction kinetics, i.e., the rate of decolorization is directly proportional to the initial concentration of the dye. The values of the apparent decolorization rate constant $k_{1}^{\prime}$ at different temperatures are shown in Table 1 . The apparent $k_{1}^{\prime}$ increased with temperature due to the increased frequency of molecular encounters between the dye and the biomass.

A first-order decolorization of dyes has been seen in studies with other fungi. For example, during decolorization of azo dye Amaranth by $T$. versicolor in a rotating biological contactor (Ramsay et al. 2006); in the decolorization of both azo and anthraquinone dyes by laccase and manganese peroxidase from T. versicolor (Champagne and Ramsay 2005); and in the decolorization of Amaranth, Reactive Black 5, Reactive Blue 19, and Direct Black 22 by alginate-immobilized $T$. versicolor (Ramsay et al. 2005).

The increase in the $k_{1}^{\prime}$ with increasing initial dye concentration at a constant temperature and with a fixed amount of the biocatalyst $\left(1.8 \times 10^{3} \mathrm{~kg} \mathrm{~m}^{-3}\right.$ in terms of volume of the pellets) suggests that the dye induced the production of decolorizing enzyme in a concentration-dependent manner. The enzyme produced remained associated with the biomass. (The biomass-free filtrate of the culture broth failed to 

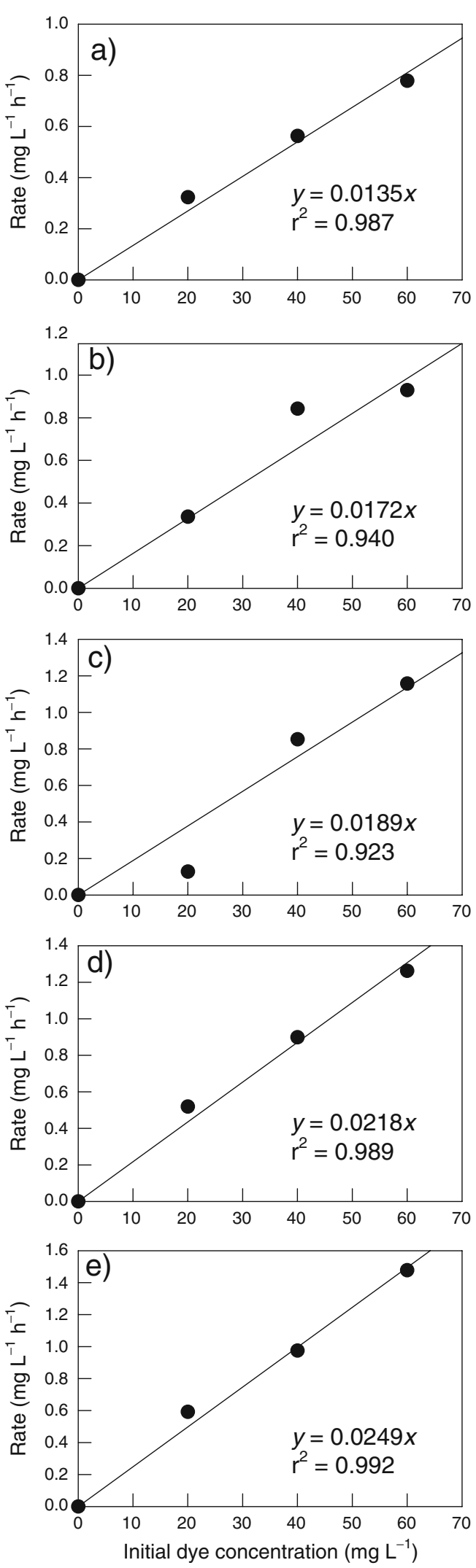

Fig. 5 The volumetric rate of dye decolorization is a linear function of initial dye concentration at different reaction temperatures: a $25^{\circ} \mathrm{C}$; b $30^{\circ} \mathrm{C}$; c $35^{\circ} \mathrm{C}$; d $40^{\circ} \mathrm{C}$; e $45^{\circ} \mathrm{C}$

decolorize the dye.) Similar findings have been reported for other white-rot fungi. For example, studies with $T$. versicolor suggest that biomassassociated components are essential for the decolorization process (Swamy and Ramsay 1999b). For example, cell-free culture filtrates of $T$. versicolor failed to decolorize Amaranth even though high concentrations of soluble laccase and manganese peroxidase were detected in the filtrate and hydrogen peroxide had been added (Swamy and Ramsay 1999b).

\subsection{Apparent Activation Energy of Decolorization}

The apparent activation energy $\left(E_{\mathrm{a}}\right)$ for the decolorization was estimated using a linearized Arrhenius plot (Fig. 6). The data fitted a straight line with a regression coefficient of 0.985 . The $E_{\mathrm{a}}$ value calculated from the slope of the line was $23 \mathrm{~kJ} \mathrm{~mol}^{-1}$. This was consistent with activation energy values for enzyme-catalyzed reactions that generally range from 16 to $84 \mathrm{~kJ} \mathrm{~mol}^{-1}$.

\subsection{Thermodynamics of Decolorization}

A van't Hoff plot of the data is shown in Fig. 7. The data fitted a straight line with a regression coefficient value of 0.979 . The apparent enthalpy $(\Delta H)$ and entropy $(\Delta S)$ were determined from the slope and the $y$ intercept of the straight line (Fig. 7). The assumptions implicit in van't Hoff analysis (i.e., a closed system at a constant pressure and volume) were

Table 1 First-order rate constants for the decolorization at various temperatures

Temperature $\left({ }^{\circ} \mathrm{C}\right) \quad$ Apparent first-order rate constant, $k_{1}{ }_{l}\left(\mathrm{~h}^{-1}\right)$

\begin{tabular}{ll}
\hline 25 & $0.0135 \pm 0.0010$ \\
30 & $0.0172 \pm 0.0029$ \\
35 & $0.0189 \pm 0.0042$ \\
40 & $0.0218 \pm 0.0016$ \\
45 & $0.0249 \pm 0.0015$
\end{tabular}




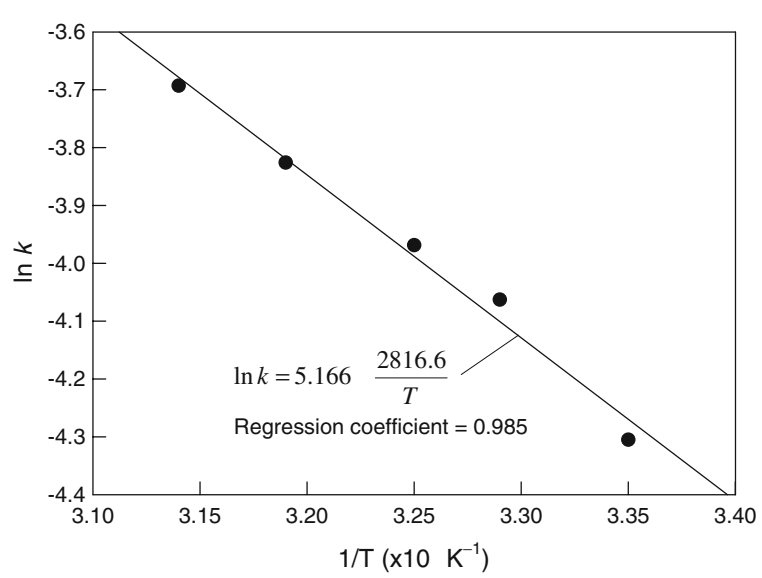

Fig. 6 Arrhenius plot for Trypan Blue decolorization by fungal pellets

applied to the decolorization reaction. The values of $\Delta H$ and $\Delta S$ were found to be 45.6 and $146 \mathrm{~J} \mathrm{~mol}^{-1}$ $\mathrm{K}^{-1}$, respectively. This implied an endothermic decolorization process that was accompanied by an increased disorder of the system as molecular structure of Trypan Blue decomposed. The positive value of $\Delta H$ explains the increasing rate of reaction with increasing temperature.

The $\Delta G$ values (Eq. 11) are listed in Table 2 for the decolorization at an initial dye concentration of $60 \mathrm{mg} \mathrm{L}^{-1}$ and various temperatures. Table 2 also presents the values of $\left(\Delta G-\Delta G^{\circ}\right)$ (Eq. 12). For the temperature range of 25 to $35^{\circ} \mathrm{C}$, the decolorization reaction does not occur spontaneously, as shown by positive $\Delta G$ values. At higher temperatures (i.e.,

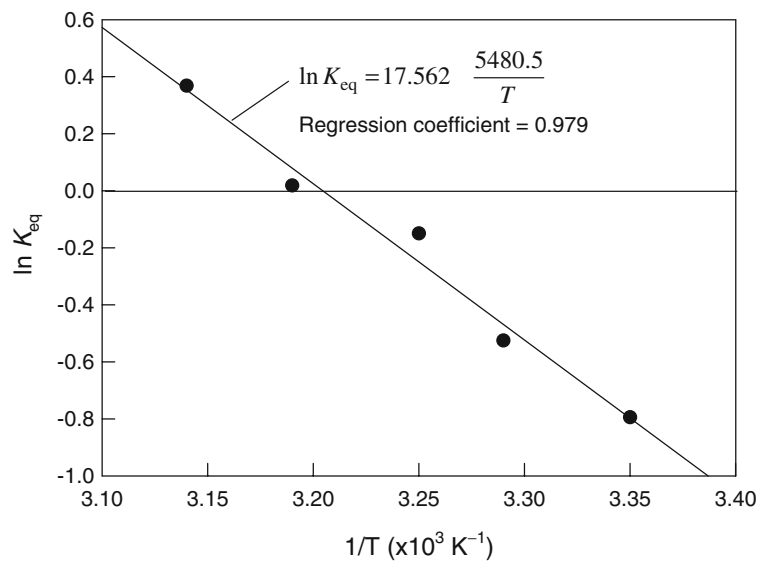

Fig. 7 Van’t Hoff plot for decolorization of Trypan Blue
Table 2 Gibbs free energy $(\Delta G)$ of decolorization at $60 \mathrm{mg}$ $\mathrm{L}^{-1}$ initial dye concentration

\begin{tabular}{lcll}
\hline$T(\mathrm{~K})$ & Apparent $K_{\text {eq }}$ & $\Delta G\left(\mathrm{~J} \mathrm{~mol}^{-1}\right)$ & $\Delta G-\Delta G^{\circ}\left(\mathrm{J} \mathrm{mol}^{-1}\right)$ \\
\hline 298.15 & 0.4518 & $+2,070$ & $-1,970$ \\
303.15 & 0.5914 & $+1,340$ & $-1,324$ \\
308.15 & 0.8614 & +610 & -382 \\
313.15 & 1.0189 & -120 & +49 \\
318.15 & 1.4459 & -850 & +975 \\
\hline
\end{tabular}

temperature $>35^{\circ} \mathrm{C}$ ), the reaction was spontaneous (i.e., negative $\Delta G$ values) (Table 2). This implies that the decolorization reaction was thermodynamically limited at temperatures of $\leq 35^{\circ} \mathrm{C}$. This assumes that mass transfer of the dye was not a limiting factor.

The value of $\left(\Delta G-\Delta G^{\circ}\right)$ indicates the difference between the energy change occurring during the reaction under the conditions used and the energy change that would occur if the reaction took place under standard conditions ( $1 \mathrm{~atm}, 298.15 \mathrm{~K}$, ideal solution). From Table 2, the energy deficit decreased for the temperature range of 25 to $35^{\circ} \mathrm{C}$ as evidenced by less negative values of $\left(\Delta G-\Delta G^{\circ}\right)$. On the other hand, an increasing energy surplus was calculated for the temperature range of $40-45^{\circ} \mathrm{C}$ (Table 2). The values of $\left(\Delta G-\Delta G^{\circ}\right)$ supported the spontaneity or non-spontaneity classification of the reaction as pointed out above.

A plot of the Gibbs free energy $(\Delta G)$ for the decolorization reaction at various temperatures is shown in Fig. 8. $\Delta G$ becomes zero at a temperature

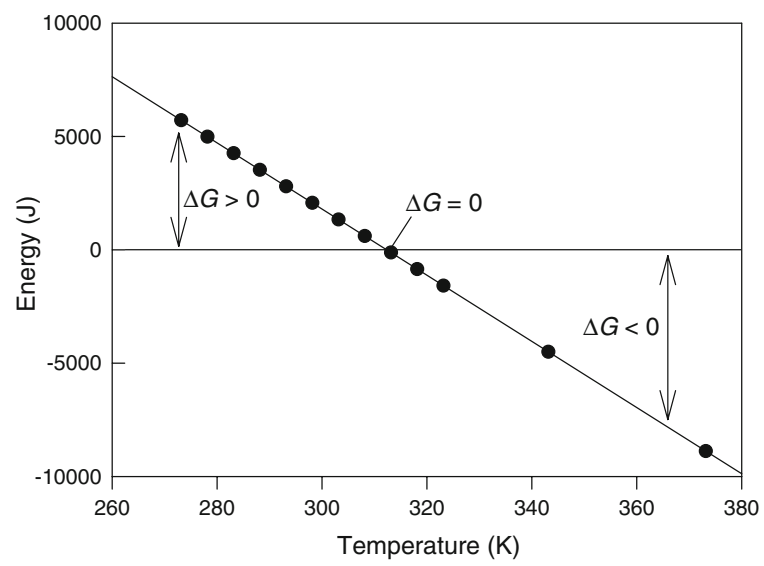

Fig. 8 Gibbs free energy change for the decolorization reaction under experimental condition as a function of temperature 
of $39.2^{\circ} \mathrm{C} . \Delta G$ is positive (i.e., non-spontaneous reaction) for lower values of temperature and it is negative for higher temperatures greater than $39.2^{\circ} \mathrm{C}$ (i.e., the decolorization reaction is favored when the temperature is above $39.2^{\circ} \mathrm{C}$ ). The regression coefficient for the line in Fig. 8 is 1.0. The equation of the line is as follows:

$$
\text { Energy }=45,600-146 T
$$

Acknowledgements The University of Malaya is thanked for financial assistance. Technical support provided by Mr. Alimin A. Karim is acknowledged.

\section{References}

Champagne, P. P., \& Ramsay, J. A. (2005). Contribution of manganese peroxidase and laccase to dye decolorization by Trametes versicolor. Applied Microbiology and Biotechnology, 69, 276-285. doi:10.1007/s00253-0051964-8.

Dursun, A. Y., \& Tepe, O. (2005). Internal mass transfer effect on biodegradation of phenol by Ca-alginate immobilized Ralstonia eutropha. Journal of Hazardous Materials, 126, 105-111. doi:10.1016/j.jhazmat.2005.06.013.

Field, J. A., de Jong, E., Costa, G. F., \& de Bont, J. A. (1992). Biodegradation of polycyclic aromatic hydrocarbons by new isolates of white rot fungi. Applied and Environmental Microbiology, 58, 2219-2226.

Michel Jr, F. C., Grulke, E. A., \& Reddy, C. A. (1992). Determination of the respiration kinetics for mycelial pellets of Phanerochaete chrysosporium. Applied and Environmental Microbiology, 58, 1740-1745.
Pointing, S. B. (2001). Feasibility of bioremediation by whiterot fungi. Applied Microbiology and Biotechnology, 57, 20-33. doi:10.1007/s002530100745.

Pointing, S. B., \& Vrijmoed, L. L. P. (2000). Decolorization of azo and triphenylmethane dyes by Pycnoporus sanguineus producing laccase as the sole phenoloxidase. World Journal of Microbiology \& Biotechnology, 16, 317-318. doi:10.1023/A:1008959600680.

Ramsay, J. A., Mok, W. H. W., Luu, Y. S., \& Savage, M. (2005). Decolorization of textile dyes by alginate-immobilized Trametes versicolor. Chemosphere, 61, 956-964. doi:10.10 16/j.chemosphere.2005.03.070.

Ramsay, J., Shin, M., Wong, S., \& Goode, C. (2006). Amaranth decoloration by Trametes versicolor in a rotating biological reactor. Journal of Industrial Microbiology \& Biotechnology, 33, 791-795. doi:10.1007/s10295-006-0117-0.

Riva, S. (2006). Laccases: blue enzymes for green chemistry. Trends in Biotechnology, 24, 219-226. doi:10.1016/j. tibtech.2006.03.006.

Shin, M., Nguyen, T., \& Ramsay, J. (2002). Evaluation of support materials for the surface immobilization and decoloration of Amaranth by Trametes versicolor. Applied Microbiology and Biotechnology, 60, 218-223. doi:10.1007/ s00253-002-1088-3.

Swamy, J., \& Ramsay, J. A. (1999a). Effects of glucose and $\mathrm{NH}^{+}{ }_{4}$ concentrations on sequential dye decoloration by Trametes versicolor. Enzyme and Microbial Technology, 25, 278-284. doi:10.1016/S0141-0229(99)00058-7.

Swamy, J., \& Ramsay, J. A. (1999b). Effects of $\mathrm{Mn}^{2+}$ and $\mathrm{NH}_{4}^{+}$ concentrations on laccase and manganese peroxidase production and Amaranth decoloration by Trametes versicolor. Applied Microbiology and Biotechnology, 51, 391396. doi:10.1007/s002530051408.

Vinod, A. V., \& Reddy, G. V. (2006). Mass transfer correlation for phenol biodegradation in a fluidized bed bioreactor. Journal of Hazardous Materials, 136, 727-734. doi:10.1016/j.jhazmat.2006.01.043.

Zollinger, H. (1987). Color chemistry-synthesis, properties and applications of organic dyes and pigments. New York: $\mathrm{VCH}$. 